\title{
Combined dural inversion with multiple burr holes for refractory chronic subdural hematoma: A case report.
}

Tuo Li ( $\nabla$ ytdzlt3528@tmu.edu.cn )

Tianjin Medical University

wei zhu

Yantai Yuhuangding Hospital

Hongying Hao

Yantai Yuhuangding Hospital

Hongguang Chen

Yantai Yuhuangding Hospital

Jianning Zhang

Tianjin Medical University

\section{Case report}

Keywords: CSDH, Recur, Bone hole, Scalp, Dural mater inversion

Posted Date: March 26th, 2021

DOI: https://doi.org/10.21203/rs.3.rs-361493/v1

License: (9) This work is licensed under a Creative Commons Attribution 4.0 International License. Read Full License 


\section{Abstract}

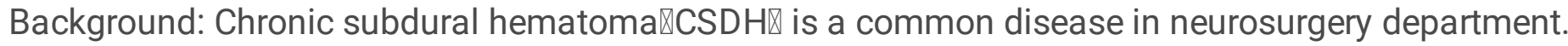
Burr-hole drainage is the main surgical treatment. And the recurrence rate is as high as $25 \%$.

Case presentation: In this case, a male patient with CSDH in the left frontotemporal parietal region underwent two drilling and drainage operations in the local hospital, but the hematoma recurred after operations. Being unable to bearing the repeated and progressive aggravation of headache, he came to our hospital for treatment. After considering the comprehensive situation, we use a new surgical method, removal of hematoma by drilling multiple holes in the lateral skull, to cure the patient.

Conclusions: we get inspirations from the therapy for moyamoya disease, combining dura mater inversion and hole burr, the scalp forms many "meat column" like structures which have powerful capability in absorption through the bone holes, so the scalp could deep into the hematoma, then the CSDH could be cured. This case Provide a new surgical method for the treatment of refractory CSDH. Key words: CSDH R ecur Bone hole Scalp Dural mater inversion

\section{Full Text}

This preprint is available for download as a PDF.

\section{Figures}



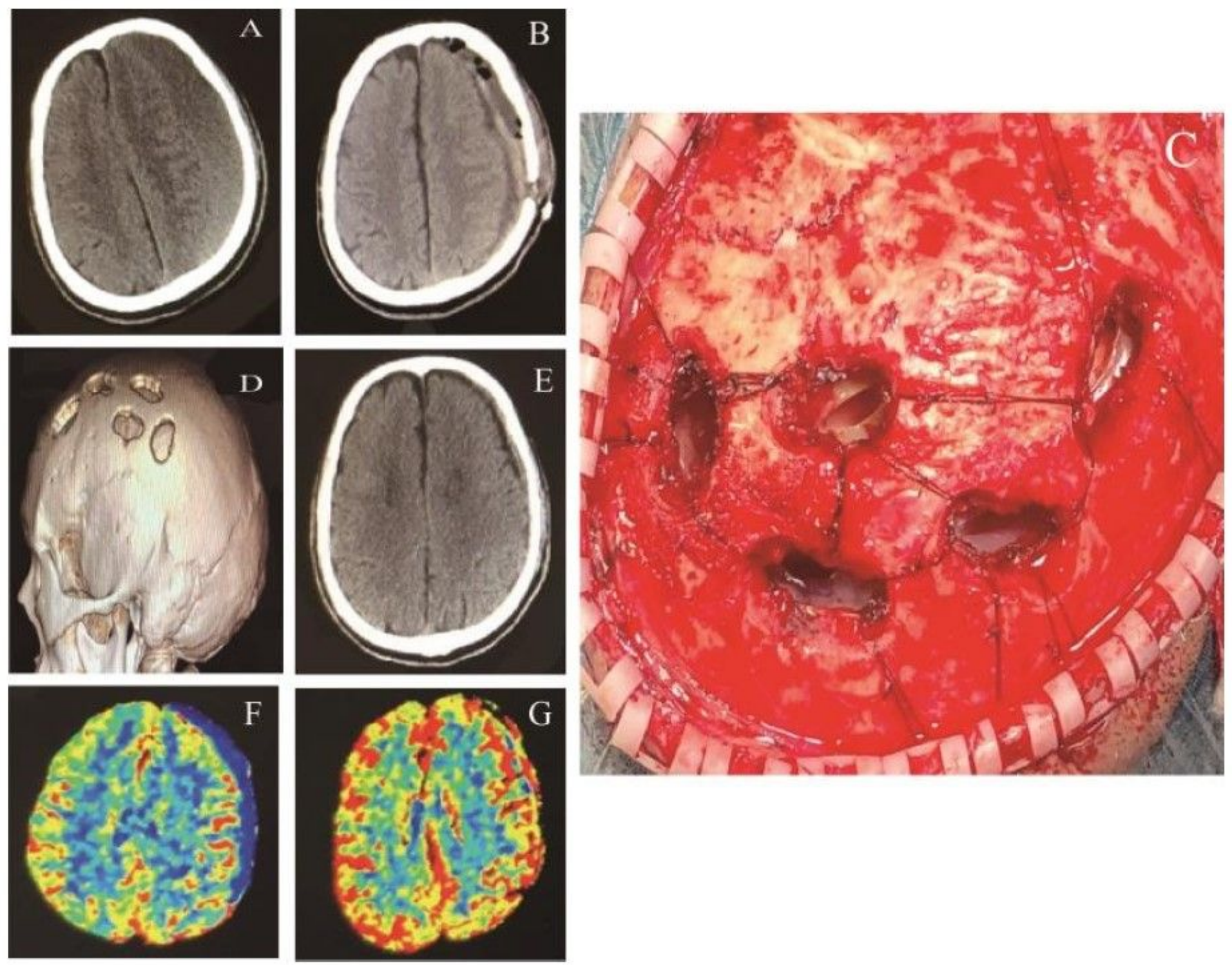

\section{Figure 1}

The CT image before and after treatment. A. CT image before operation. B and D. CT image after operation. $C$ the operation image of bone holes and dura mater inversion. E. CT image a month after operation. $\mathrm{F}$ and $\mathrm{G}$ cranial blood infusion before and after operation. 


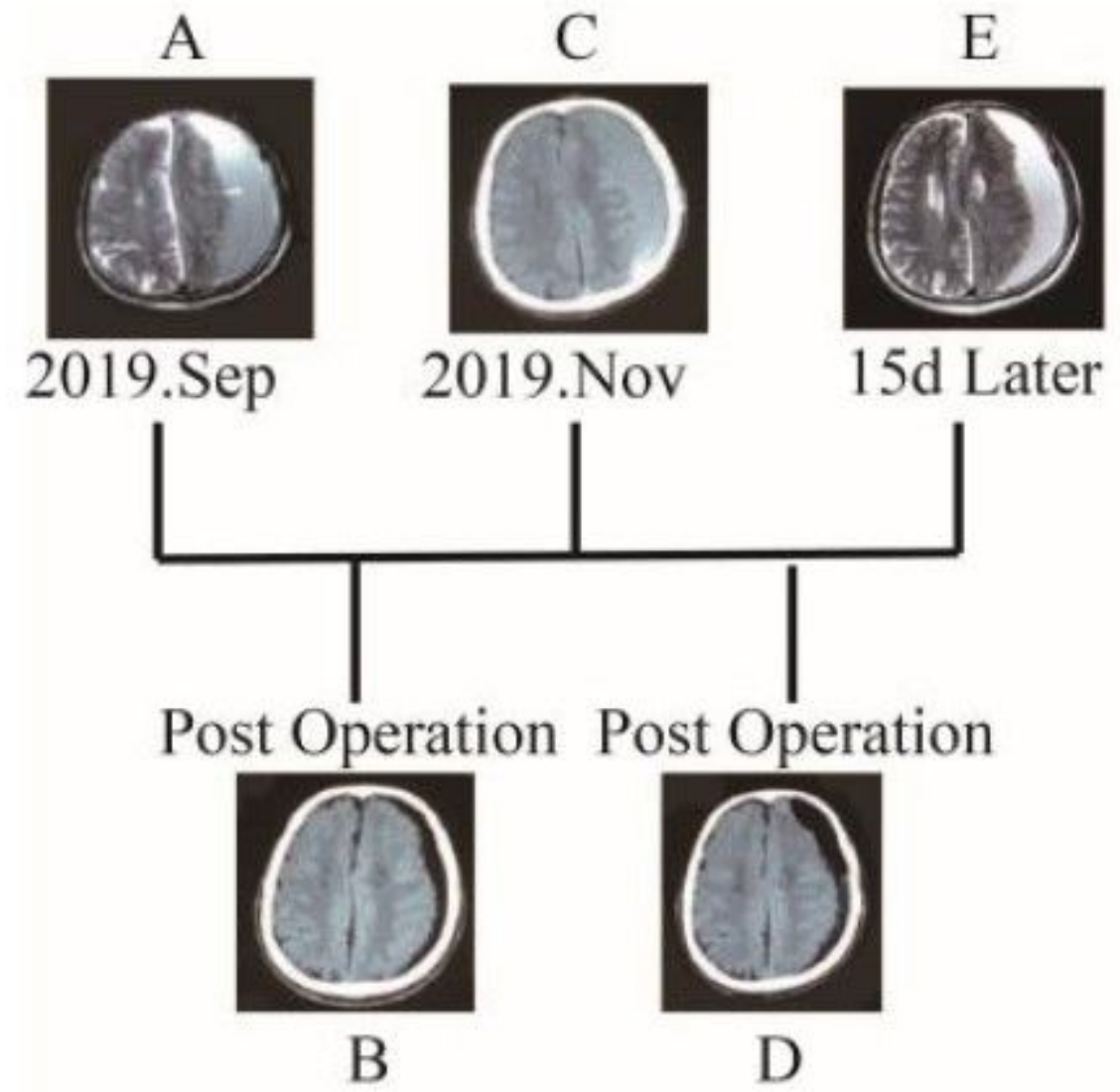

Figure 2

CT image of hematoma within 4 months. 